\title{
Elaboración de un modelo evolutivo híbrido de algoritmos genéticos y redes neuronales artificiales para dosificaciones de mezclas de concreto reforzadas con fibras metálicas
}

\author{
Development of a Hybrid Evolutionary Model of Genetic Algorithms and Artificial Neural Networks for \\ Metal Fiber and Reinforced Concrete Mixture Dosage
}

\begin{abstract}
Luis Octavio González Salcedo (1), Aydee Patricia Guerrero Zúñiga ${ }^{(2)}$, Silvio Delvasto Arjona ${ }^{(3)}$, Adrián Luis Ernesto Will (4)
(1) Doctor en Ingeniería de Materiales. Profesor Asociado, Facultad de Ingeniería y Administración, Universidad Nacional de Colombia Sede Palmira. Palmira, Colombia. Grupo de Investigación en Materiales y Medio Ambiente. logonzalezsa@unal.edu.co.

(2) Doctor of Philosophy in Civil Engineering. Profesora Titular, Facultad de Ingeniería, Universidad del Valle. Cali, Colombia. Grupo de Investigación en Ingeniería Sísmica, Eólica, Geotécnica y Estructural. patricia.guerrero@correounivalle.edu.co

(3) Doctor en Nuevos Materiales y sus Tecnologías de Fabricación, Profesor Titular, Facultad de Ingeniería, Universidad del Valle, Cali, Colombia. Grupo de Materiales Compuestos. silvio.delvasto@correounivalle.edu.co

${ }^{(4)}$ Doctor en Matemáticas. Profesor Adjunto, Facultad de Ciencias Exactas y Tecnología - Universidad Nacional de Tucumán, Facultad Regional de Tucumán - Universidad Tecnológica Nacional. Tucumán, Argentina. Grupo de Investigación de Tecnologías Avanzadas de Tucumán. adrian.will.01@gmail.com
\end{abstract}

Recibido 15 de agosto de 2015. Modificado 30 de noviembre de 2015. Aprobado 9 de diciembre de 2015.

DOI: http://dx.doi.org/10.16924/riua.v0i43.874

\section{Palabras clave}

Algoritmos genéticos, dosificaciones de mezclas de concreto, modelo evolutivo, redes neuronales artificiales.

\section{Resumen}

En un ambiente computacional, un modelo evolutivo es desarrollado para proponer dosificaciones de mezclas de concretos reforzados con fibras metálicas, para solicitudes de resistencia a la compresión. El modelo es híbrido al incluir un sistema de dosificación basado en algoritmos genéticos y un sistema de estimación de propiedades basado en redes neuronales artificiales. Los resultados obtenidos son comparados con un sistema de dosificaciones reportado experimentalmente. Estas comparaciones muestran una aproximación en el proceso de simulación. Además, por las características del modelo, se considera de aporte en la tecnología del concreto.

\section{Key words}

Genetic Algorithms, Concrete Mixture Dosages, Evolutionary Model, Artificial Neural Networks.

\begin{abstract}
An evolutionary model is developed in a computing environment to propose metal fiber reinforced concrete mixture dosages for compressive strength applications. The model is hybrid as it includes both a dosage system based on genetic algorithm and a properties prediction system based on artificial neural networks. The results obtained are compared with experimentally reported dosages set, and the comparisons show an approximation in the simulation process. Given the characteristics of the model, it is considered a contribution to concrete technology.
\end{abstract}

\section{ITRODUCCIÓN}

Esta investigación aborda la estimación de dosificaciones de mezclas de concreto reforzado con fibras metálicas usando Algoritmos Evolutivos, basados en la técnica de los Algoritmos Genéticos (AG). Se requiere en algunos concretos de un comportamiento de desempeño correspondiente a un ambiente aplicado, y hoy en día, el progreso de la tecnología del concreto permite hacer que el concreto encuentre estos requerimientos. Sin embargo, aunque hay manera de optimizar matemáticamente el problema en muchos criterios, los cuales están representados por funciones objetivos, el problema de decisión es multivariado y una amplia variedad de dosificaciones como soluciones son posibles (Siddique \& Khan, 2011). 
El problema de la dosificación es un problema complejo que no se puede resolver por los métodos habituales de programación lineal y no lineal. Adicionalmente, la dosificación de las mezclas de concreto se realiza en el caso de los concretos de resistencia normal, usando protocolos suficientemente probados, pero para concretos de alta resistencia, la dosificación es en general aún del tipo de prueba y error, existiendo algunos pocos métodos sugeridos en la literatura especializada (Martínez, 2008). Para ambos casos, es decir, concretos de resistencia normal y de alta resistencia, los métodos de diseño usados conllevan a un único diseño de mezclas (Sánchez, 2000; ASOCRETO, 2010). Por otro lado, la determinación adecuada de fibra (cantidad, morfología, diámetro y longitud), como refuerzo del concreto, frecuentemente ha sido definida de manera experimental, estando su optimización limitada por los recursos a utilizar, como la cantidad de fibra, el costo de ensayos de laboratorio, y el tiempo, entre otros (Holschemacher et al., 2006). El requerimiento de adiciones minerales como las cenizas volantes, el humo de sílice y la escoria de altos hornos en concretos de prestaciones específicas conlleva también a que sus dosificaciones sean determinadas de manera experimental (Siddique \& Khan, 2011).

Los AG son algoritmos de optimización, aprendizaje y búsqueda basados en el mecanismo de la selección natural y la genética natural, y se ha comprobado su efectividad en aplicaciones en el campo de la ingeniería, especialmente para problemas de combinación. Dado que la dosificación de las mezclas de concreto no es solamente un tipo de problema de combinación, sino también de optimización combinatoria y compleja que involucra variables numéricas y cualitativas, es entonces una buena razón aplicar en este caso los AG, a la par de que la dosificación del concreto posiblemente induzca a la aparición de cálculos en funciones difíciles de evaluar o de altos errores de medición (Noguchi et al, 2003). Setién et al. (2006) ratifican el problema en la determinación de las propiedades del concreto a partir de las dosificaciones de la mezcla, y sugieren la introducción de técnicas basadas en el uso de Redes Neuronales Artificiales (RNA), dada la complejidad e incertidumbre de los factores allí involucrados.

\section{Metodología}

Para el desarrollo de la investigación se elabora un Modelo Evolutivo Híbrido, denominado así en razón de la estructura del Modelo Evolutivo basado en AG que incorpora un Modelo Neuronal basado en RNA. El AG se usa como un Sistema de Dosificación, mientras la RNA, diseñada como un Sistema de Estimación, en este caso como componente de la estructura del sistema de dosificación, se usa como Función de Estimación. El procedimiento metodológico, la definición de sus elementos y sus operadores genéticos, las bases fundamentales, y los detalles de construcción de un Modelo Evolutivo basado en AG son determinados por Goldberg (1989). En su funcionamiento típico, los AG requieren de elementos iniciales como una población posible y potencial de soluciones, la aplicación de operaciones genéticas, la determinación de una función de evaluación o de adaptación al medio, y el mecanismo de evaluación a esa adaptación o supervivencia mediante una penalización. En adición, se requiere incorporar operaciones de restricción y ajuste, para aproximar el Modelo Evolutivo al problema que se quiere simular.

\section{POBLACIÓN INICIAL}

Se plantea un conjunto que describa el sistema de dosificación en el diseño de mezclas de concreto reforzado con fibras metálicas. La dosificación del concreto consiste en la determinación de las proporciones en que han de mezclarse los componentes que lo constituyen. Dado que el concreto está constituido por diversos materiales, no existe una única

\begin{tabular}{|c|c|c|}
\hline Variable & $\begin{array}{c}\text { Unidad } \\
\text { (variable } \\
\text { cuantitativa) }\end{array}$ & $\begin{array}{c}\text { Atributo } \\
\text { (variable } \\
\text { cualitativa } \\
\text { representada } \\
\text { como variable } \\
\text { discreta) }\end{array}$ \\
\hline Contenido de cemento ${ }^{1}$ & $\mathrm{Kg} / \mathrm{m}^{3}$ & - \\
\hline Tipo de cemento & - & Tipos I, II, III, IV, V \\
\hline Contenido de humo de sílice ${ }^{1}$ & $\mathrm{Kg} / \mathrm{m}^{3}$ & - \\
\hline Contenido de cenizas volantes ${ }^{1}$ & $\mathrm{Kg} / \mathrm{m}^{3}$ & - \\
\hline Contenido de escoria ${ }^{1}$ & $\mathrm{Kg} / \mathrm{m}^{3}$ & - \\
\hline Contenido de agua $^{1}$ & $\mathrm{Kg} / \mathrm{m}^{3}$ & - \\
\hline $\begin{array}{l}\text { Contenido de agente retenedor } \\
\text { de agua }^{1}\end{array}$ & $\mathrm{Kg} / \mathrm{m}^{3}$ & - \\
\hline $\begin{array}{l}\text { Tipo de agente retenedor de } \\
\text { agua }\end{array}$ & - & $\begin{array}{l}\text { Plastificante, } \\
\text { Superplastificante }\end{array}$ \\
\hline Contenido de arena ${ }^{1}$ & $\mathrm{Kg} / \mathrm{m}^{3}$ & - \\
\hline $\begin{array}{l}\text { Origen de procedencia de la } \\
\text { arena }\end{array}$ & - & Río, Cantera \\
\hline Contenido de Grava $^{1}$ & $\mathrm{Kg} / \mathrm{m}^{3}$ & - \\
\hline $\begin{array}{l}\text { Origen de procedencia de la } \\
\text { grava }\end{array}$ & - & Río, Cantera \\
\hline Origen geológico de la grava & - & $\begin{array}{l}\text { Perfil litológico } \\
\text { I, II, III }\end{array}$ \\
\hline Tamaño máximo del agregado & $\mathrm{mm}$ & - \\
\hline $\begin{array}{l}\text { Contenido de agente inclusor } \\
\qquad \text { de aire }{ }^{1}\end{array}$ & $\mathrm{Kg} / \mathrm{m}^{3}$ & - \\
\hline Contenido de fibra metálica ${ }^{1}$ & $\mathrm{Kg} / \mathrm{m}^{3}$ & - \\
\hline Tipo de fibra metálica & - & Tipo I, II, III y IV \\
\hline Longitud de la fibra & $\mathrm{mm}$ & - \\
\hline Relación de aspecto de la fibra & Adimensional & - \\
\hline \multicolumn{3}{|c|}{$\begin{array}{c}{ }^{1} \text { Los contenidos de los materiales están expresados en } \mathrm{Kg} \text { por } \mathrm{m}^{3} \text { de } \\
\text { mezcla de concreto. }\end{array}$} \\
\hline
\end{tabular}

Tabla 1. Variables relevantes del problema de dosificación, consideradas en el Sistema de Dosificación. Fuente: Elaboración Propia. 


\begin{tabular}{|c|c|c|c|c|}
\hline Variable & $\begin{array}{c}f^{\prime} c \leq 30 \\
M P a\end{array}$ & $\begin{array}{l}30<f^{\prime} c \leq \\
60 \mathrm{MPa}\end{array}$ & $\begin{array}{l}60<f^{\prime} c \leq \leq \\
95 \mathrm{MPa}\end{array}$ & $\begin{array}{c}f^{\prime}>95 \\
\mathrm{MPa}\end{array}$ \\
\hline Cemento & $250-400$ & $300-600$ & $350-700$ & $420-800$ \\
\hline $\begin{array}{l}\text { Tipo de } \\
\text { Cemento }^{1}\end{array}$ & \multicolumn{4}{|c|}{$0-1$} \\
\hline Humo de Sílice ${ }^{2}$ & $0-80$ & $0-120$ & $0-120$ & $0-160$ \\
\hline $\begin{array}{c}\text { Cenizas } \\
\text { Volantes }^{3}\end{array}$ & $0-120$ & $0-180$ & $0-180$ & $0-240$ \\
\hline Escoria $^{4}$ & $0-160$ & $0-240$ & $0-240$ & $0-320$ \\
\hline Agua $^{5}$ & $125-200$ & $140-280$ & $155-315$ & $175-335$ \\
\hline Fluidizante $^{6}$ & $0-5$ & $0-7,5$ & $0-8,75$ & $0-10$ \\
\hline $\begin{array}{c}\text { Tipo de } \\
\text { Fluidizante }^{7}\end{array}$ & \multicolumn{4}{|c|}{$0-1$} \\
\hline Arena & $600-900$ & $600-1100$ & $450-850$ & $450-1000$ \\
\hline Procedencia ${ }^{8}$ & \multicolumn{4}{|c|}{$0-1$} \\
\hline Grava & $800-1250$ & $800-1300$ & $\begin{array}{l}800 \\
1350\end{array}$ & $800-1400$ \\
\hline Procedencia $^{9}$ & \multicolumn{4}{|c|}{$0-1$} \\
\hline $\begin{array}{l}\text { Tamaño } \\
\text { Máximo }\end{array}$ & \multicolumn{4}{|c|}{$9,57-19,05$} \\
\hline $\begin{array}{c}\text { Origen } \\
\text { Geológico }^{10}\end{array}$ & \multicolumn{4}{|c|}{$0-1$} \\
\hline $\begin{array}{l}\text { Inclusor de } \\
\text { Aire }^{11}\end{array}$ & $0-0,8$ & $0-1,2$ & $0-1,4$ & $0-1,6$ \\
\hline Fibra & $\begin{array}{c}15,72- \\
78,60\end{array}$ & $\begin{array}{l}31,44 \\
157,20\end{array}$ & $\begin{array}{l}31,44 \quad- \\
157,20\end{array}$ & $\begin{array}{l}39,30- \\
157,20\end{array}$ \\
\hline Tipo de Fibra ${ }^{12}$ & \multicolumn{4}{|c|}{$0-1$} \\
\hline Longitud & \multicolumn{4}{|c|}{$30-60$} \\
\hline $\begin{array}{l}\text { Relación de } \\
\text { Aspecto }\end{array}$ & \multicolumn{4}{|c|}{$50-80$} \\
\hline \multicolumn{5}{|c|}{$\begin{array}{l}{ }^{1} \text { Se generan cinco clases mutuamente excluyentes; }{ }^{2} \text { hasta un } 20 \% \text { de reemplazo; } \\
{ }^{3} \text { hasta un } 30 \% \text { de reemplazo; }{ }^{4} \text { hasta un } 40 \% \text { de reemplazo; }{ }^{5} \text { control de la relación } \\
\text { W/B } \approx 0,5 ;{ }^{6} \text { hasta una dosificación del } 1,25 \% \text { por peso de cementante; }{ }^{7} \text { se } \\
\text { generan dos clases mutuamente excluyentes; }{ }^{8} \text { se generan dos clases mutuamente } \\
\text { excluyentes; }{ }^{9} \text { se generan dos clases mutuamente excluyentes; }{ }^{10} \text { se generan tres } \\
\text { clases mutuamente excluyentes; }{ }^{11} \text { hasta una dosificación del } 0,2 \% \text { por peso de } \\
\text { cementante; }{ }^{12} \text { se generan cuatro clases mutuamente excluyentes. }\end{array}$} \\
\hline
\end{tabular}

Tabla 2. Límites mínimo y máximo, en la matriz de límites, para definir la construcción de la población inicial en concretos fibrorreforzados, para cuatro rangos de resistencia a la compresión f'c. Fuente: Elaboración Propia.

dosificación posible para obtener una resistencia a la compresión f’c (se toma esta propiedad mecánica, en razón al cumplimiento en las especificaciones de construcción), sino que hay un rango de dosificaciones posibles que responden a esa misma propiedad mecánica. Por esta razón, la población inicial se define a partir de dos aspectos: las variables a considerar dentro de la dosificación y los límites del rango para cada variable. Con respecto al primer aspecto, las variables consideradas como relevantes del problema de dosificación son mostradas en la Tabla 1.

Se crea una matriz de límites para cada variable considerada en la dosificación, en la cual se definen el valor mínimo

\begin{tabular}{|c|c|c|c|}
\hline Variable & Variable & Variable & Variable \\
\hline Cemento: 5 & Agua: 2,5 & Grava: 5 & Fibra: 7,86 \\
\hline Tipo de Cemento: 0 & Fluidizante: 0,1 & $\begin{array}{c}\text { Procedencia: } \\
0\end{array}$ & $\begin{array}{l}\text { Tipo de } \\
\text { fibra: } 0\end{array}$ \\
\hline Humo de Sílice: 5 & $\begin{array}{c}\text { Tipo de } \\
\text { Fluidizante: } 0\end{array}$ & $\begin{array}{c}\text { Tamaño } \\
\text { Máximo: } 0,25\end{array}$ & Longitud: 5 \\
\hline Cenizas Volantes: 5 & Arena: 5 & $\begin{array}{c}\text { Origen } \\
\text { Geológico: } \\
0-1\end{array}$ & $\begin{array}{c}\text { Relación de } \\
\text { aspecto: } 5\end{array}$ \\
\hline Escoria: 5 & Procedencia: 0 & $\begin{array}{l}\text { Inclusor de } \\
\text { Aire: } 0,01\end{array}$ & \\
\hline
\end{tabular}

Tabla 3. Valores asignados a cada variable en el Vector Distancia Fuente: Elaboración

\begin{tabular}{|r|l|}
\hline Arquitectura: & $\begin{array}{l}\text { Multicapa, Feedforward - } \\
\text { Blackpropagation. }\end{array}$ \\
\hline $\begin{array}{r}\text { Conjunto de } \\
\text { Entrenamiento: }\end{array}$ & 611 vectores de información. \\
\hline $\begin{array}{r}\text { División Interna de } \\
\text { Datos: }\end{array}$ & $\begin{array}{r}\text { Aleatoria, en tres conjuntos: } \\
\text { Entrenamiento, Prueba y } \\
\text { Validación. }\end{array}$ \\
\hline Capa de Entrada: & $\begin{array}{l}30 \text { neuronas representando } \\
12 \text { variables cuantitativas y 18 } \\
\text { variables cualitativas. }\end{array}$ \\
\hline Capas Ocultas: & $\begin{array}{l}\text { Tres capas ocultas con 25, 15 y } \\
5 \text { neuronas, respectivamente. }\end{array}$ \\
\hline Capa de Salid a: & $\begin{array}{l}1 \text { neurona representando la } \\
\text { variable a estimar, f'c. }\end{array}$ \\
\hline $\begin{array}{r}\text { Algoritmo de de } \\
\text { Aprendizaje: }\end{array}$ & $\begin{array}{l}\text { Levenberg - Marquardt } \\
\text { (Lourakis, 2005) }\end{array}$ \\
\hline $\begin{array}{l}\text { Indicador de } \\
\text { Desempeño: }\end{array}$ & Error Cuadrado Total \\
\hline
\end{tabular}

Tabla 4. Características de la RNA seleccionada como Sistema de Estimación de la resistencia a la compresión f'c, usada como función de estimación, en el AG propuesto.

Fuente: Elaboración Propia.

y el valor máximo de cada una de ellas, basados en trabajos realizados con anterioridad sobre optimización de componentes de dosificaciones de concreto (Will et al., 2008; Yeh, 2009; Chen \& Wang, 2010), y la recopilación de 601 diseños de mezclas en un sistema de dosificación de datos experimentales reportados en la literatura especializada (González, 2014). Se muestra en la Tabla 2, la matriz de límites para la población inicial en concretos para cuatro rangos de resistencia a la compresión f'c.

Para la aplicación de los operadores genéticos se requiere la definición de una función distancia, con el fin de identificar en los posibles individuos seleccionados (soluciones 
seleccionadas) si existen diferencias significativas entre ellas desde el punto de vista de sus componentes genéticos, es decir, la configuración del “cromosoma”. La función distancia se muestra en la Tabla 3.

\section{FunCión DE EsTIMACIÓN}

Como función de estimación, se usa un sistema de predicción basado en un modelo neuronal artificial, entrenado para la estimación de la resistencia a la compresión, dada una dosificación de acuerdo con las variables de entrada consideradas en la Tabla 1. El modelo neuronal artificial consistió de una RNA, con arquitectura multicapa, que responde a un problema de decisión de dimensión 30 (resultante de las 19 variables mostradas en la Tabla 1), del cual considera 12 variables referidas a las cantidades de los materiales y 18 variables referidas a atributos o cualidades de los materiales, y cuyo desempeño se evaluó con el coeficiente de correlación lineal R. Para el entrenamiento de la RNA, se construyó un conjunto de entrenamiento conformado por 611 diseños de mezclas conducentes a una resistencia a la compresión f'c, recopilados de la literatura especializada (González, 2014). Además, se emplearon diferentes arquitecturas (capas ocultas y números de neuronas en las capas ocultas) y diferentes algoritmos de aprendizaje feedforward - blackpropagation. Como lenguaje de programación del algoritmo de la RNA se usa MATLAB ${ }^{\circledR}$ \& SIMULINK ${ }^{\circledR}$ para ambiente WINDOWS ${ }^{\circledR}$ (The MathWorks Inc, 2008), y la codificación se ha basado en el Neural Network Toolbox del lenguaje mencionado (Beale et al., 2012). La RNA seleccionada como sistema de estimación correspondió a una red multicapa con las características mostradas en la Tabla 4. El Factor de Correlación R es mostrado en la Figura 1.
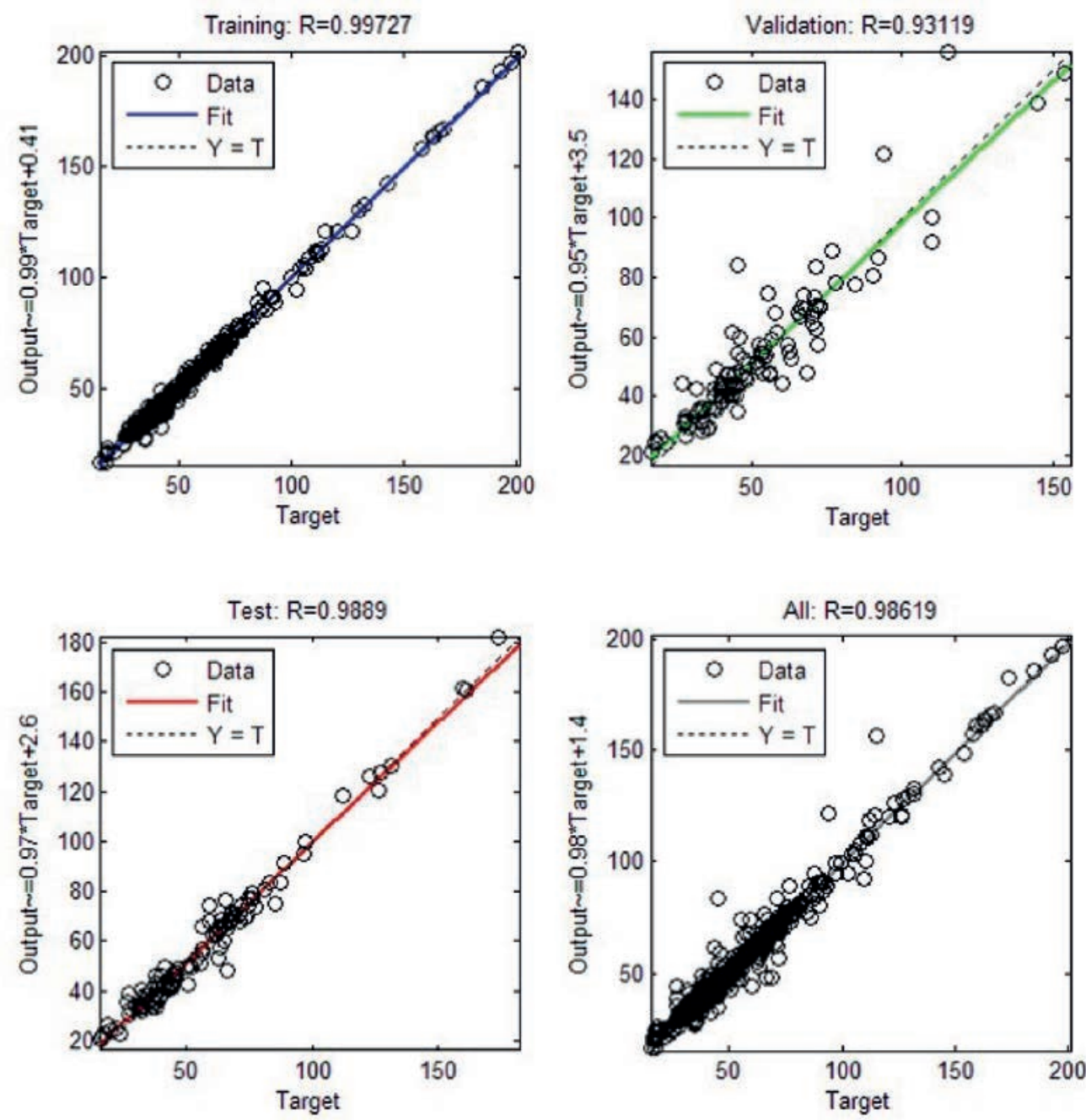

Figura 1. Factor de Correlación Lineal, para la RNA usada como función de estimación, para los conjuntos de Entrenamiento, Prueba, Validación, y con Total de Datos Fuente: Elaboración propia, 2015. 


\section{RESTRICCIONES Y FUNCIONES DE AJUSTE}

El objetivo de los AG en esta investigación es proponer la dosificación en mezclas de concreto reforzados con fibras metálicas que satisfarán un valor de resistencia a la compresión requerido. De igual manera, se respeta un número importante de restricciones propias del proceso de diseño de la mezcla, como se hace en los procedimientos protocolarios de esta actividad. Se plantean funciones para realizar las siguientes restricciones y funciones de ajustes (ASOCRETO, 2010): Control Volumétrico (1,00 m³), Relación Agua Total / Cementante $(0,40-0,60)$, limitación en el uso de aditivos químicos, limitación en el reemplazo del cemento por materiales cementantes y limitación en el tamaño máximo del agregado grueso debido al tamaño de la fibra metálica empleada.

\section{Función Objetivo y Función de Penalización}

Se usa una función de evaluación para múltiples criterios: la resistencia a la compresión, el asentamiento del cono de Abrams, el control volumétrico, la relación agua total/cementante, la limitación en el uso de los aditivos químicos, la limitación en el reemplazo del cemento por materiales cementantes, el tamaño máximo del agregado como función del diámetro de la fibra, y el Asentamiento del Cono de Abrams como función de la cantidad de agua, del tamaño máximo del agregado y del tipo del agregado, esquematizado en la representación de la Ecuación 1: máximo y del tipo de agregado. La penalización es realizada a partir de una función continua formada por segmentos de recta en forma de embudo, la cual en los extremos tiene un valor de altura, disminuyendo hacia el centro hasta un valor de cero. La función tiene como objetivo premiar los valores en el segmento central con cero y castigar con valores progresivamente mayores hacia los extremos.

\section{Operadores Genéticos}

Como operadores de selección o reproducción (Gil, 2006) se usan Ruleta y Muestreo Universal Estocástico; como operadores de cruce (Gil, 2006) se usan Cruce Uniforme, Heurístico y Aritmético; por Recombinación Intermedia y para Permutación se usa Mapeamiento Parcial y de Ciclo, y como operador de mutación (Davis, 1991) se usa No Uniforme y Uniforme.

\section{ARQUITECTURA DEL MODELO EVOLUTIVO}

En el laboratorio computacional del Grupo de Aplicaciones de Matemática e Inteligencia Artificial de la Facultad de Ciencias Exactas y Tecnología - Universidad Nacional de Tucumán, localizada en San Miguel de Tucumán (Argentina) se elaboró un modelo básico de Algoritmos Genéticos, para el problema de dosificación en mezclas de concreto no

$$
\begin{gathered}
f(\text { sol })=f_{\text {Penalización }}\left[\left(f^{\prime}{ }_{C r}-\text { Objetivo }\right),(\text { Slump - Objetivo }),\left(1-\text { Vol }_{\text {Mezcla }}\right),\right. \\
\left(0,5-\frac{W_{T}}{B}\right),\left(0,00625-\frac{H W R}{B}\right),\left(0,001-\frac{A E}{B}\right),\left(0,1-\frac{S F}{B}\right),\left(0,15-\frac{F A}{B}\right),\left(0,2-\frac{G B F S}{B}\right), \\
\left.f_{T M A}\left(V_{\rho}, D_{f}\right)-T M A, f_{T M A}\left(L_{f}\right)-T M A, f_{\text {Slump }}\left(T M A, W_{T}\right)-\text { Slump }\right]
\end{gathered}
$$

Donde f(sol) es la función de evaluación para cada solución representada en una dosificación propuesta; sol es la dosificación propuesta, $\mathrm{f}_{\text {Penalización }}$ es una función de penalización que permite calificar (premiar o castigar el criterio en la solución), $\mathrm{f}_{\mathrm{Cr}}$ es la resistencia a la compresión que aportará la solución, Slump es el asentamiento del cono de Abrams que aportará la solución, el término Objetivo corresponde para cada propiedad el valor buscado, $\mathrm{Vol}_{\text {Mezcla }}$ es el volumen aportado por la dosificación de la mezcla, $\mathrm{W}_{\mathrm{T}}$ es el contenido total de agua, B es el contenido de material cementante, HWR es el contenido de agente retenedor de agua, $\mathrm{AE}$ es el contenido de agente inclusor de aire, SF es el contenido de humo de sílice, FA es el contenido de cenizas volantes, GBFS es el contenido de escoria, $f_{\text {TMA }}$ es el tamaño máximo del agregado en función del volumen de fibra y del diámetro de la fibra, $\mathrm{V}_{\mathrm{f}}$ y $\mathrm{D}_{\mathrm{f}}$ respectivamente, $\mathrm{y}$ de la longitud de fibra $L_{f}$, TMA es el tamaño máximo del agregado, $\mathrm{y} \mathrm{f}_{\text {SLump }}$ es el asentamiento del Cono de Abrams en función del contenido de agua total $\mathrm{W}_{\mathrm{T}} \mathrm{y}$ del tamaño reforzadas, cuyos resultados fueron analizados usando indicadores de comparación entre un sistema de dosificación de datos experimentales reportados y el de los resultados obtenidos por el modelo mencionado (González et al., 2009). Este modelo fue modificado, complementado y adaptado al problema de dosificaciones de mezclas en concretos reforzados con fibras, en las instalaciones de la Universidad del Valle, localizada en Santiago de Cali (Colombia); las adaptaciones correspondieron a la incorporación de las características específicas relacionadas con la generación de la población inicial, la función de estimación, las restricciones y las funciones de ajustes, la función objetivo y de penalización, y los operadores genéticos, mencionados con anterioridad. Para el modelo evolutivo basado en algoritmos genéticos se elaboraron un conjunto de programas de computador usando como lenguaje de programación para su escritura MATLAB ${ }^{\circledR}$ para ambiente Windows ${ }^{\circledR}$ (The MathWorks Inc, 2008); la codificación se basó en el Genetic Algorithm Toolbox del lenguaje mencionado (The MathWorks Inc, 2004). El conjunto de 
programas computacionales se estructuró en una compleja arquitectura consistente de siete módulos de primer orden, que comprende un programa principal para dar inicio a los algoritmos genéticos y seis programas auxiliares; veinticinco módulos de segundo orden, dispuestos a manera de librería para el programa principal; y, los módulos del primer orden, cuya funcionalidad es realizar todas las operaciones simuladas computacionalmente de un proceso de evolución.

\section{RESULTADOS Y DISCUSIÓN}

El modelo evolutivo basado en algoritmos genéticos se ejecutó para solucionar dosificaciones de mezclas de concreto reforzados con fibras, con requerimientos de resistencia a la compresión de 21, 30, 40, 50, 60, 70, 80, 90 y 100 MPa. A manera de ejemplo, en este documento se muestran algunos resultados para la simulación realizada, considerando en el diseño de la mezcla cemento Tipo I: superplastificante como agente retenedor de agua, arena procedente de río, grava procedente de cantera y perfil litológico I y fibra metálica tipo Hooke-end de longitud $30 \mathrm{~mm}$ y relación de aspecto 80. Para cada resistencia mencionada, se consideraron tres tipos de adiciones minerales a saber: en la primera, se consideró cenizas volantes, en la segunda se consideraron cenizas volantes y escoria, y en la tercera se consideró humo de sílice (Figura 2).
Para el análisis de los resultados, se presentan algunos indicadores de comparación entre el sistema de dosificación de los datos experimentales reportados y el de los resultados obtenidos por el Modelo Evolutivo (Figura 3). De la misma manera, se toman como indicadores de comparación relaciones entre los materiales cementantes y el volumen de fibra empleados en las dosificaciones (Figura 4).

Los resultados de estas comparaciones muestran que los parámetros del material mencionados tienen una similitud de tendencia para ambos sistemas, de la siguiente manera:

- Con respecto al contenido de material cementante (total de cemento, humo de sílice, cenizas volantes y escoria), (Fig. 3), se observa en ambos sistemas de dosificación que este parámetro se incrementa para mayores requerimientos de resistencia, coincidiendo con Will et al., 2008, y Yeh, 2009.

- Con respecto a la relación del contenido de agua total/ contenido del material cementante (W/B), (Fig. 3), se observa en ambos sistemas de dosificación que este parámetro se comporta con la relación clásica f'c vs W/B (ASOCRETO, 2010), en la cual a medida que se incrementa el valor del parámetro, la resistencia a la compresión disminuye, coincidiendo con Will et al., 2008, y Yeh, 2009.

- Con respecto a las distribuciones de frecuencia para la relación entre los contenidos de adiciones minera-
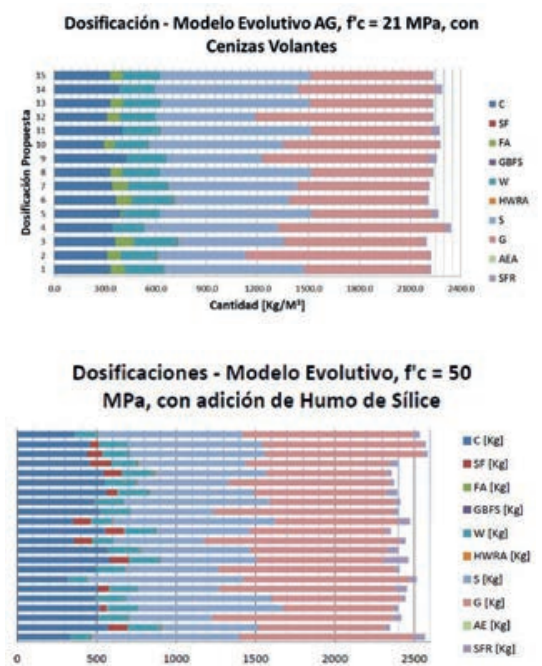

Dosificaciones - Modelo Evolutivo AG, $f^{\prime} c=$ $80 \mathrm{MPa}$, con adición de Cenizas Volantes
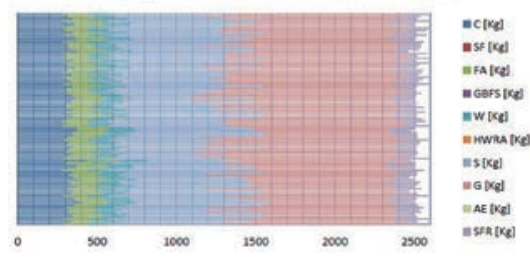
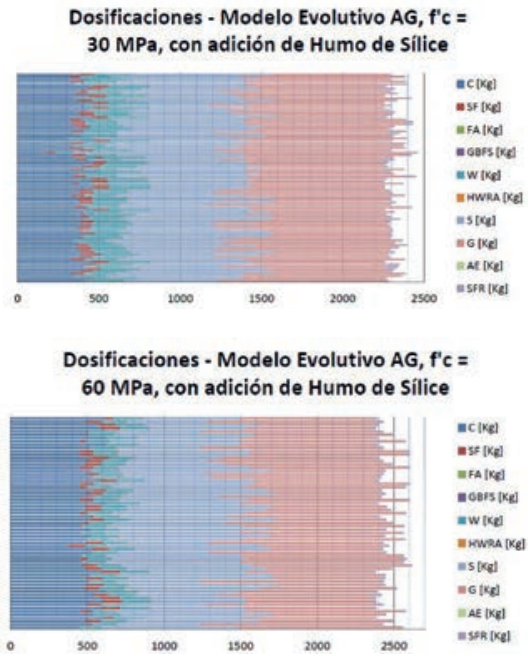

Dosificaciones - Modelo Evolutivo AG, $\mathrm{f}^{\prime} \mathrm{C}=$ $90 \mathrm{MPa}$, con adición de Cenizas Volantes y Escoria

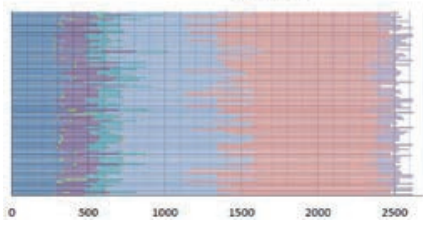

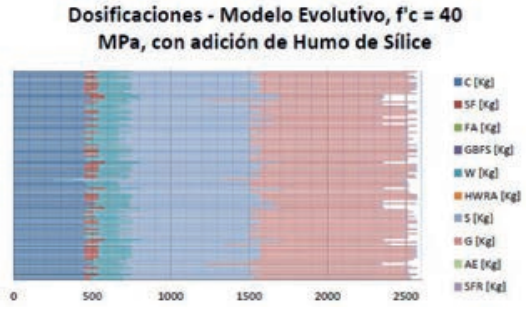
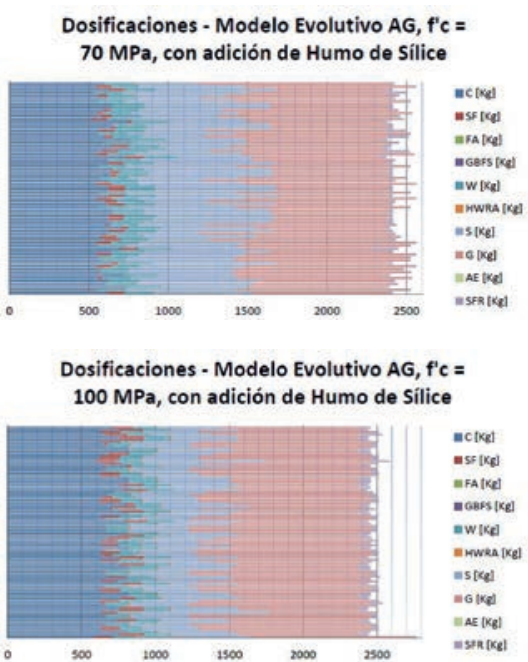

Figura 2. Dosificaciones obtenidas para casos específicos de resistencia a la compresión f'c. Fuente:Elaboración propia, 2015. 

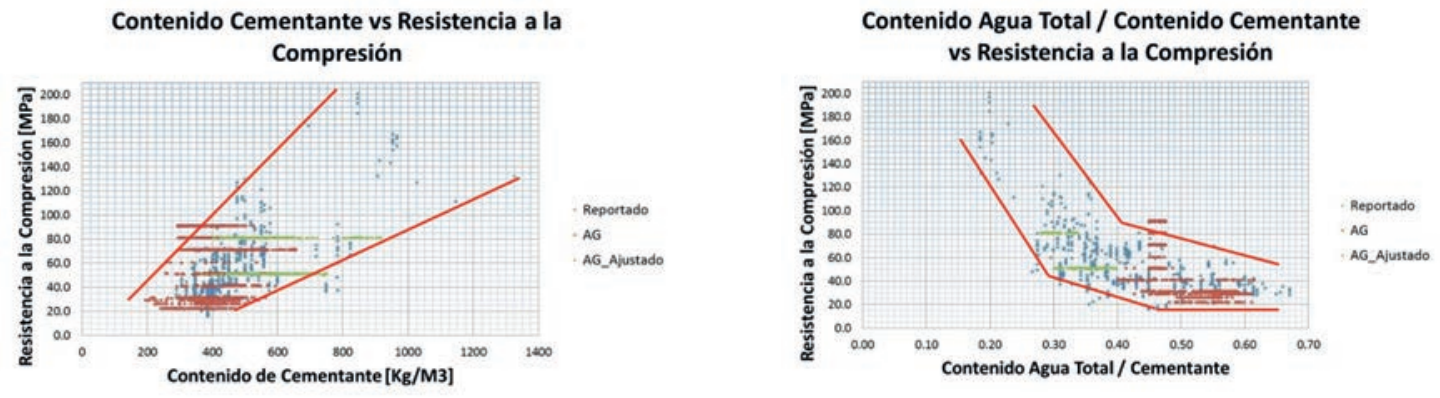

Figura 3. Indicadores de comparación entre el sistema de dosificación de los datos experimentales reportados y el de los resultados obtenidos por el Modelo Evolutivo. Fuente: Elaboración propia, 2015.
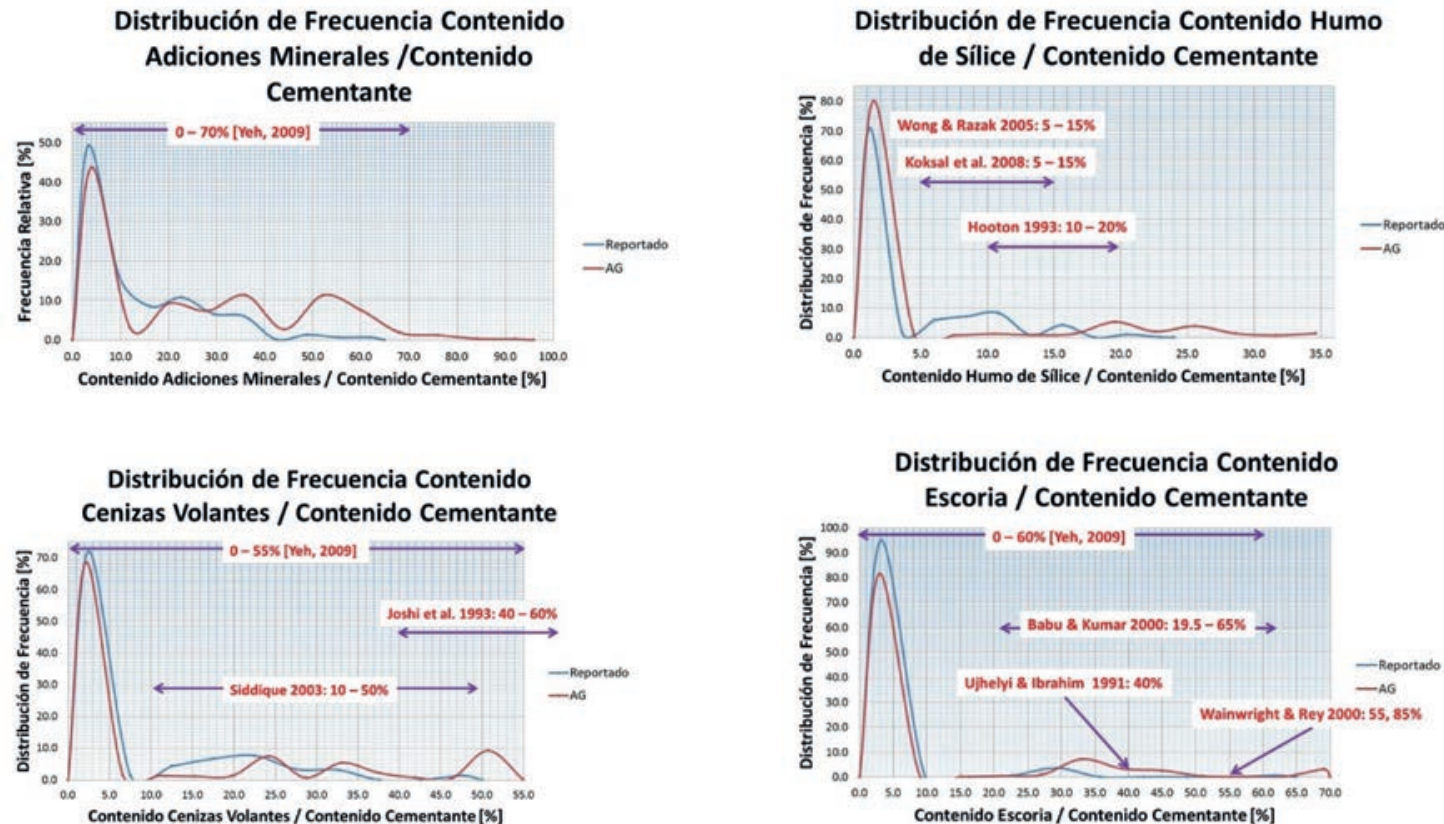

Distribución de Frecuencia Fracción de Volumen de Fibra

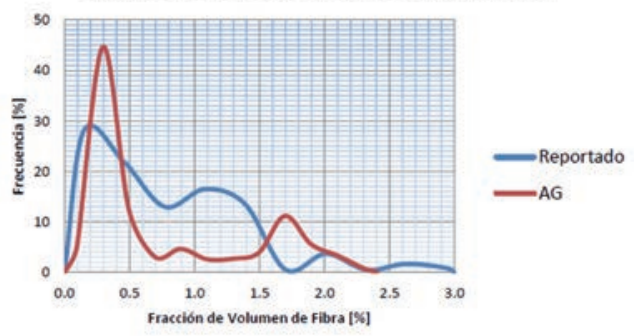

Figura 4. Indicadores de comparación basados en relaciones entre el sistema de dosificación de los datos experimentales reportados y el de los resultados obtenidos por el modelo evolutivo. Fuente: Elaboración Propia.

les (contenidos de humo de sílice, cenizas volantes y escoria) total y específicos y el contenido del material cementante (Fig. 4), se observa en ambos sistemas de dosificación que este parámetro tiene tendencias similares. En particular se hacen las siguientes apreciaciones:

- El sistema de dosificación propuesto por el AG se caracteriza por emplear reemplazos del cemento por cantidades controladas de adiciones minerales, de una manera similar a como se realiza en el sistema de dosificación reportado.

- El sistema de dosificaciones propuesto por el AG sigue una tendencia similar en cuanto a los porcentajes de reemplazo para cada adición mineral, mostrando un número importante de dosificaciones para reemplazos entre el 12-15\% 
para humo de sílice, el 20-22\% para cenizas volantes, y alrededor del 25\% para escoria, lo cual concuerda con los valores máximos sugeridos en la literatura, para cada caso (Siddique \& Khan, 2011).

- El sistema de dosificaciones propuesto coincide en reemplazos específicos reportados en otras investigaciones, como se detalla en la Figura 4, coincidiendo con Ujhelyi \& Ibrahim, 1991, Hooton 1993, Joshi et al., 1993, Babu \& Kumar, 2000, Wainwright \& Rey, 2000, Wong \& Razak, 2005, Koksal et al., 2008, y Yeh, 2009.

Con respecto a otros modelos evolutivos para el mismo objetivo, se reportan en la literatura modelos basados en algoritmos genéticos, estrategias evolutivas o programación evolutiva, para encontrar soluciones en la dosificación de mezclas de concretos sin/con adiciones minerales, y sin/con aditivos químicos (uso de plastificantes principalmente), $\mathrm{y}$ usando funciones de estimación u otras técnicas de inteligencia artificial, referidas a las cantidades de la materia prima a usar (Noguchi et al., 2003, Özgan \& Oztürg, 2007, Will et al. 2008, Yeh, 2009, Chen \& Wang, 2010). Se resalta que no existen desarrollos sobre estos modelos evolutivos y en particular híbridos, para encontrar soluciones en la dosificación de mezclas de concretos reforzadas con fibras.

\section{CONCLUSIONES}

El modelo genera un conjunto de posibles dosificaciones, las cuales son evaluadas en la estimación de la resistencia a la compresión y categorizadas de acuerdo con el resultado obtenido, para ser escogidas como las mejores propuestas de dosificación acorde a la propiedad mecánica requerida. Las propuestas son sometidas a modificaciones, usando operaciones genéticas, para encontrar otras dosificaciones que cumplan con el mismo objetivo de alcanzar la propiedad mecánica. Los resultados muestran una alta cantidad de propuestas de dosificaciones de mezclas de concreto reforzadas con fibras, las cuales evidencian indicadores de similitud en la tendencia de diversos parámetros del material, así como una similitud en el contenido de materiales relevantes de comparación como el contenido del material cementante y del contenido de agua total, al ser comparada con un sistema de dosificaciones experimentales reportadas. Los resultados del modelo evolutivo permiten trazar las siguientes conclusiones:

- $\quad$ Se abre una futura agenda de investigación con las propuestas de dosificaciones de las mezclas, con la cual puede realizarse un análisis interesante para conformar clasificaciones como concretos con adiciones simples, binarias y terciarias, entre otras.

- $\quad$ El modelo evolutivo puede ser ajustado para usar en sus funciones objetivo y de penalización (ajustando fPenalización en la Ec.1) y la incorporación de las correspondientes funciones de estimación. Además, la estimación de otras propiedades de tal manera que la obtención de propuestas de dosificaciones que se realicen para objetivos de criterios múltiples, es decir, que satisfagan no solo valores de resistencia a la compresión, sino también otras propiedades tanto en estado fresco (asentamientos) como en estado endurecido (propiedades mecánicas y de durabilidad), abre también una futura agenda de interés para el campo de la tecnología del concreto.

- $\quad$ La consideración de variables tanto de tipo cuantitativa como cualitativa, como componentes de la mezcla, en similitud a las variables de entrada en el entrenamiento de las redes neuronales artificiales, posibilitan el diseño de un modelo híbrido, conformado por un modelo evolutivo cuya función de estimación es realizada por el modelo neuronal.

Se concluye que el modelo evolutivo desarrollado y expuesto en la presente investigación, en razón a sus características, constituye un aporte importante en la tecnología del concreto, específicamente en la dosificación de mezclas de concretos reforzados con fibras metálicas.

\section{REFERENCIAS}

Asociación Colombiana de Productores de Concreto -ASOCRETO. (2010). Tecnología y Propiedades. Colección Básica del Concreto. Tomo 1. Bogotá: Instituto del Concreto, ASOCRETO.

Babu, K.G. \& Kumar, V.S.R. (2000). Efficiency of GGBS in concrete. Cement Concrete Research, 30(7), 1031-1036. doi:10.1016/S0008-8846(00)00271-4

Beale, M.H., Hagan, M.T. \& Demuth, H.B. (2012). Neural Network ToolboxTM, User's Guide. Natick: The MathWorks Inc.

Chen, L. \& Wang, T-S. (2010). Modeling Strength of HighPerformance Concrete Using and Improved Grammatical Evolution Combined with Macrogenetic Algorithm. Journal of Computing in Civil Engineering, 24(3), 281288. doi: 10.1061/(ASCE)CP.1943-5487.0000031

Davis, L.D. (Ed). (1991). The Handbook of Genetic Algorithms. New York:Van Nostrand Reinhold.

Gil, L. N. (2006). Algoritmos Genéticos. Universidad Nacional de Colombia Sede Medellín. Medellín, Noviembre, 65p.

Goldberg, D.E. (1989). Genetic Algorithms in Search: Optimization and Learning. USA: Addison-Wesley Publishing Co., Reading.

González S., L.O.; (2014). Diseño de mezclas de concreto reforzado con fibras metálicas y de polipropileno, usando inteligencia artificial. (Tesis inédita de doctorado) Universidad del Valle, Santiago de Cali, Colombia. 
González, S., L.O., Torres A., J. \& WILL, A.L.E. (2009, octubre). Evaluación de diseños de mezclas de concreto obtenidos usando Inteligencia Artificial basada en Algoritmos Genéticos. Ponencia presentada en V Congreso Internacional de Materiales, Memorias. Santiago de Cali, Colombia.

Holschemacher, K., Müller, T. \& Fischer, A. (2006, august). Effect of fibre type on properties of steel fibre reinforced concrete. Ponencia presentada en The Tenth East AsiaPacific Conference on Structural Engineering \& Construction (EASEC-10). Asian Institute of Technology. Bangkok, Thailand.

Hooton, , R.D. (1993). Influence of silica fume replacement of cement on physical properties and resistance to sulfate attack freezing and thawing, and alkali-silica reactivity. ACI Materials Journal, 90(2), 143-152.

Joshi, R.C., Lohtia, R.P. \& Salam, M.A. (1993). High strength concrete with high volumes of Canadian sub-bituminous coal ash. Ponencia presentada en Third International Symposium on Utilization of High Strength Concrete. Lillachammer, Norway.

Köksal, F., Altun,, F., Yigit, I. \& Súahin, Y. (2008). Combined effect of silica fume and Steel fibre on the mechanical properties of high strength concretes. Construction Building Materials, 22(8), 1874-1880. doi:10.1016/j. conbuildmat.2007.04.017

Lourakis, M.A. (2005). A brief description for the LevenbergMarquardt Algorithm implemented by levmar. Heraklion: Institute of Computer Science, Foundation for Research and Technology - Hellas (FORTH).

Martínez P., D. (2008). Hormigones de altas prestaciones. (Proyecto Fin de Carrera Ingeniería Técnica Civil: Arquitectura Técnica), Universidad Politécnica de Cartagena, Cartagena, España.

Noguchi, T., Maruyama, I. \& Kanematsu, M. (2003, may). Performance based design for concrete mixture with multi-optimizing Genetic Algorithm. Ponencia presentada en The 11th International Congress on the Chemistry of Cement (ICCC): “Cement's contribution to the development in the 21st Century”. Cement \& Concrete Institute. Durban, South Africa.

Özgan, E. \& Öztürk, A. (2007). Optimization of the Hardened Concrete Properties with GA and LP. Journal of Applied Sciences, 7(24), 3918-3926.
Sánchez D.G., D. (2000). Tecnología del Concreto y del Mortero. $2^{\mathrm{a}}$ edición. Bogotá: Biblioteca de la Construcción Bhandar Editores.

Siddique, R. (2003). Effect of fine aggregate replacement with class $\mathrm{F}$ fly ash on the mechanical properties of concrete. Cement Concrete Research, 33(4), 539-547. doi:10.1016/S0008-8846(02)01000-1

Siddique,, R. \& Khan, M.I.; (2011). Supplementary Cementing Materials. New York: Springer. Engineering Materials Collection.

Sietén, J., Carrascal, I.A., Figueroa, J.F. \& Polanco, J.A. (2003). Aplicación de una red neuronal artificial a la dosificación de hormigones premezclados. Materiales de Construcción, 53(270), 5-19. doi: 10.3989/mc.2003.v53. i2 270.270

The Mathworks Inc. (2004). Genetic Algorithm and Direct Search Toolbox for use with MATLAB®, User's Guide. Version 1. Natick: The MathWorks Inc.

The Mathworks Inc. (2008). MATLAB® \& SIMULINK® Release 2008a, Installation Guide for Windows. Natick: The MathWorks Inc.

Ujhelyi, J.E. \& Ibrahim, A.J. (1991). Hot weather concreting with hydraulic additives. Cement Concrete Research, 21(2-3), 345-354. doi:10.1016/0008-8846(91)90015-A

Wainwright, P.J. \& Rey, N. (2000). The influence of ground granulated blast furnace slag (GGBS) additions and time delay on the bleeding of concrete. Cement Concrete Composites, 22(4), 253-257. doi:10.1016/S09589465(00)00024-X

Will, A., Folino, P., Bálzamo, H. \& Etse, G. (2008). Acerca de la conveniencia del uso de algoritmos genéticos como herramienta para la dosificación de hormigones. Mecánica Computacional, 27(12), 865-880.

Wong, H.S. \& Razak, H.A. (2005). Efficiency of calcined kaolin and silica fume as cement replacement material for strength performance. Cement Concrete Research, 35(4), 696-702. doi:10.1016/j.cemconres.2004.05.051

Yeh, I.C. (2009). Optimization of concrete mix proportioning using a flattened simplex-centroid mixture design and neural networks. Engineering with Computers, 25(2), 179-190. doi: 10.1007/s00366-008-0113-2 